\title{
A Longitudinal Study of Sexuality Training for the Interdisciplinary Rehabilitation Team
}

\author{
Patricia Fronek $^{1,4}$, Melissa Kendall ${ }^{2,3,4}$, Susan Booth ${ }^{2}$, Ellen Eugarde ${ }^{5}$ and Timothy Geraghty \\ (1) School of Human Services and Social Work, Griffith University, Logan Campus, University Drive, Meadowbrook, \\ Brisbane, QLD, 4131, Australia \\ (2) Transitional Rehabilitation Program, Queensland Spinal Cord Injury Service, Brisbane, Australia \\ (3) Acquired Brain Injury Outreach Service, Princess Alexandra Hospital, Brisbane, Australia \\ (4) Griffith Health Institute, Griffith University, Brisbane, Australia \\ (5) Spinal Injuries Unit, Queensland Spinal Cord Injury Service, Brisbane, Australia
}

\author{
Patricia Fronek \\ Email: p.fronek@griffith.edu.au
}

\begin{abstract}
Discipline specific and multidisciplinary training programs have been developed to provide practitioners working in health and disability fields with various combinations of knowledge, skills, attitudes and practitioner comfort important for such discussions. There is little, however, which demonstrates the long term effectiveness of such training programs. The current study is a 2-year follow-up to a randomized controlled trial that examined the effectiveness of a sexuality training program for rehabilitation practitioners working within spinal cord injury rehabilitation. Using both questionnaire-based and qualitative data, findings suggest that training can have longlasting effects on practitioner knowledge, comfort and attitudes in addressing sexuality issues with clients. Training that is developed around the individualized needs of practitioners and the settings within which they work may be particularly effective. Future research is needed to explore the applicability of this type of training to other rehabilitation and healthcare settings.
\end{abstract}

Keywords Sexuality - Training - Interdisciplinary - Spinal cord injury - Australia

\section{Introduction}

Sexual function and intimate relationships, fundamental to the human condition, are radically affected by spinal cord injury (SCI). Yet these are not topics that are always discussed effortlessly between rehabilitation practitioners and the people they seek to assist [1-4]. The importance of addressing sexuality issues and multidisciplinary practitioner competence in managing such discussions with people with spinal cord injuries have 
long been highlighted in the literature $[\underline{1}, \underline{3}, \underline{5}-\underline{7}]$. Discipline specific and multidisciplinary training programs have been developed to provide practitioners working in health and disability fields with various combinations of knowledge, skills, attitudes and practitioner comfort important for such discussions [ $\underline{7}-\underline{13}]$. There is little, however, which demonstrates the long term effectiveness of such training programs.

Earlier studies tended to focus on discipline specific rather than on interprofessional or multidisciplinary training $[\underline{4}, \underline{14}-17]$. The influence of practitioner training on knowledge and attitudes in the field of intellectual disability [10] and on knowledge, comfort and attitudes in the field of spinal cord injury rehabilitation has been shown [ 7 ]. These studies, however, used small sample sizes, were not randomized controlled trials and, in the case of Rose and Holmes [10], did not measure longitudinal change.

More recently, a few studies have demonstrated the effectiveness of sexuality training courses for rehabilitation practitioners working in a range of disability and health fields $[\underline{8}, \underline{12}, \underline{13}]$. Variations in study design, the length and content of training programs and participants' fields of practice are noted. Simpson et al. [토] addressed multidisciplinary practitioner sexuality training in neurological rehabilitation in New Zealand using a prospective controlled pre- and post-test evaluation of 74 participants in the treatment group and 25 in the control group. Evaluation of the 2-day workshop showed changes in knowledge, comfort and attitudes, some of which were maintained at 6 months despite reporting a low response rate at this measurement point. Increased clinical activity related to sexuality rehabilitation suggesting some translation to practice was self-reported in the 6 months to follow up. Post et al. [12] provided training to a sample of 283 multidisciplinary participants from two rehabilitation centres in the Netherlands. Education was delivered in shorter training sessions (2-3 h) designed for and delivered to discipline specific groups. This study did not utilize a control group and addressed sexuality training in a discipline specific context rather than adopting an interprofessional approach. A Dutch adaptation of the KCAASS (Knowledge, Comfort, Approach and Attitudes towards Sexuality Scale) [12, 18] was used as the outcome measure at pre-test and post-test and at 3-4 months follow-up. Improvements were noted in all domains though changes were not consistent between disciplines. Post et al. [ㅌ1], like Simpson [ $\underline{13}$ ], also reported high attrition at 3-4 month follow-up.

In Fronek et al.’s [] randomized controlled trial, a 1-day sexuality training program, developed from a needs assessment [19], was delivered to multidisciplinary practitioners working in an Australian spinal cord injuries rehabilitation service, a model spanning hospital and community-based settings [20]. Eighty-six practitioners were randomized into experimental and control groups. An interprofessional learning environment was promoted in the training sessions. Significant improvements were noted in practitioner knowledge regarding sexuality and spinal cord injuries, practitioner comfort (general and personal) and attitudes towards sexuality issues in the treatment group where no significant change was noted in the control group. These domains were measured by the KCAASS [18] immediately pre- and post-education and at 3 month follow-up. Improvements were maintained at 3 months. The control group was also educated after 3 months to ensure equity of access to educational opportunities. This paper presents findings from a 2-year follow-up study conducted to investigate whether changes in the original experimental group noted at 3 month follow-up were maintained in the long term. Further, the study sought to determine whether the control group who received training following the 3 month follow-up experienced similar improvements in knowledge, comfort and attitudes to those previously identified in the experimental group.

\section{Method}

This study is a 2-year follow-up to a randomized controlled trial that aimed to measure changes in knowledge, comfort and attitudes following a 1-day training program. The study aimed to determine whether reported gains in the experimental group at 3 months were maintained at 2 years and whether the control group, who received training in the 4th month, had similarly improved knowledge, comfort and attitudes at the 2 year period. The 2 year follow-up used a combination of both quantitative and qualitative techniques. 


\section{Participants}

Participants were practitioners from the Queensland Spinal Cord Injuries Service (QSCIS) which is made up of the Spinal Injuries Unit (SIU), Transitional Rehabilitation Team (TRP) and the Spinal Outreach Team (SPOT), the organizations that previously participated in the randomized controlled trial. Only individuals who completed all three previous assessments were selected for inclusion $(\mathrm{N}=84)$. A total of 37 multidisciplinary practitioners from the original 89 participants previously randomized to experimental and control groups completed the questionnaire, representing $41.6 \%$ of the original sample and $44 \%$ of the targeted sample. The sample of 37 included 23 nurses, 1 medical practitioner, 3 allied health staff and 10 community staff. Attrition was attributable to staff turnover, maternity and annual leave taken at the data collection time, and some individuals $(\mathrm{n}=6)$ who declined to participate. This was particularly evident within hospital-based staff, especially nurses where only 23 of the original 63 nurses (36\%) participated and only 6 out of 32 nurses within the control group participated (19\%) at 2 year follow-up. There were however no significant differences between those who participated at 2 year follow-up and those in the attrition group with respect to scores on the KCAASS at any measurement point.

\section{Intervention}

The intervention, a 1-day workshop developed for the needs of an interdisciplinary audience, has been previously described []․

\section{Measures}

The quantitative component of the follow-up involved the administration of the KCAASS [18] to both experimental and control groups. This measure has been used consistently throughout the research to date [요, $\underline{18}$, 19] with demonstrated construct validity and internal consistency. Four summary scores are produced for the scale, namely Knowledge (/56), Comfort (/104), Approach (/20) and Attitude (/20). Higher scores represent greater knowledge and skills. The Comfort, Approach and Attitude Subscales are reverse coded for calculating summary scores.

The qualitative component of the study consisted of a focus group discussion, conducted as part of an interdisciplinary in-service across the three wings of the service involved in the randomized controlled trial. In addition, participants were asked to provide written feedback to open-ended questions included on the KCAASS itself. These questions asked about attitudes, benefits of the training and possible future needs.

\section{Procedure}

Approval was sought and obtained from the relevant human research ethics committee to carry out the 2 year follow-up study which was not part of the originally submitted and approved protocol. Information sheets and consent forms were provided to participants. Given the previous familiarity of the participants with the nature of the research, participation was considered low risk. Participants were able to withdraw at any time from the research and their confidentiality was maintained through the use of non-identifying information.

The focus group was conducted prior to administration of the KCAASS (including open ended questions). For the focus group, participants were initially presented with a summary of the original findings from the randomized controlled trial and then were asked to comment about the benefits and challenges of the training. Written notes were taken during the focus group by a scribe.

The KCAASS was administered approximately 4 months later within the confines of the ordinary working day, under workplace conditions as there was no collective gathering within which it could be administered. 
Administration was coordinated by the research team across each of the disciplines within both hospital and community settings. Data collection stretched over a period of 1 month to capture individuals working across shifts and to ensure that short periods of annual leave did not prevent participation in the follow-up.

\section{Data Analysis}

Descriptive statistics were used to illustrate central tendency and variability on each of the subscales of the KCAASS at follow-up. Skewed distributions, as found in previous studies, were identified for the Attitude subscale, prompting the need for non-parametric analysis of the data. Between-group comparisons of the experimental and control group were conducted using Mann-Whitney $U$ test and within-group comparisons over time were conducted using Friedman's test and the Wilcoxon signed ranks test for post hoc comparisons.

Qualitative analysis was conducted using a pragmatic approach to thematic analysis outlined by Aronson [리]. Scribed data taken from the focus group was combined with written comments made in the 2 year follow-up completion of the open-ended questions on the KCAASS. Existing experiences were themed based on the questions asked and additional experiences and themes were identified throughout the analysis process. Themes were units derived from patterns such as "conversation topics, vocabulary, recurring activities, meanings, feelings” (p. 131) [22]. Themes that emerged during analysis were then reconsidered as a whole to form a comprehensive picture of their collective experience.

\section{Results}

The final sample for the 2 year follow-up consisted of 37 individuals, namely 25 from the original experimental group and 12 from the control group. This sample included 23 nurses, 1 medical practitioner, 3 allied health staff and 10 community staff. There were insufficient numbers within each discipline to allow cross-discipline comparisons in the analysis.

While both experimental and control groups were separated for the original randomized controlled trial, both groups had received the training at 2 year follow-up. As such, it was anticipated that the groups would not differ on the subscales of the KCAASS. There was no missing data for the 2 year follow-up.

As hypothesized, between-group comparisons revealed that there were no significant differences on any of the subscales between the experimental and control groups at the 2 year follow-up. This contrasts with significant differences that existed at 3 month follow-up, prior to the provision of training to the control group. At that point, the groups differed significantly on the Knowledge subscale $(Z=-3.328, p=0.001)$, with the experimental group scoring higher.

Within-group comparisons conducted to explore change over time from the initial administration of the scales as part of the randomized trial to that at 2 year follow-up revealed no significant changes over the entire study time in the control group but significant change in the experimental group on the Knowledge $\left(?^{2}=38.58, p<0.001\right)$, Comfort $\left(?^{2}=19.64, p<0.001\right)$ and Approach $\left(?^{2}=21.54, p<0.001\right)$ subscales. Significant change over time was noted in both the experimental $\left(?^{2}=12.30, p=0.006\right)$ and control $\left(?^{2}=10.77, p=0.013\right)$ groups on the Attitude subscale. Figures $\underline{1}, \underline{2}, \underline{3}$ and $\underline{4}$ highlight the trajectories of knowledge, comfort, approach and attitude over the 2 year study period. 


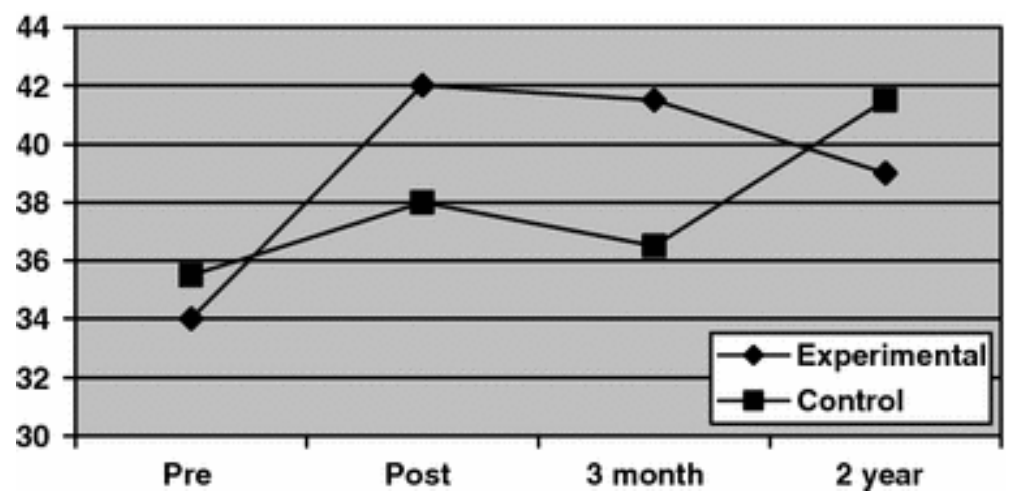

Fig. 1 Two year trajectories on the Knowledge subscale of the KCAASS for experimental and control groups

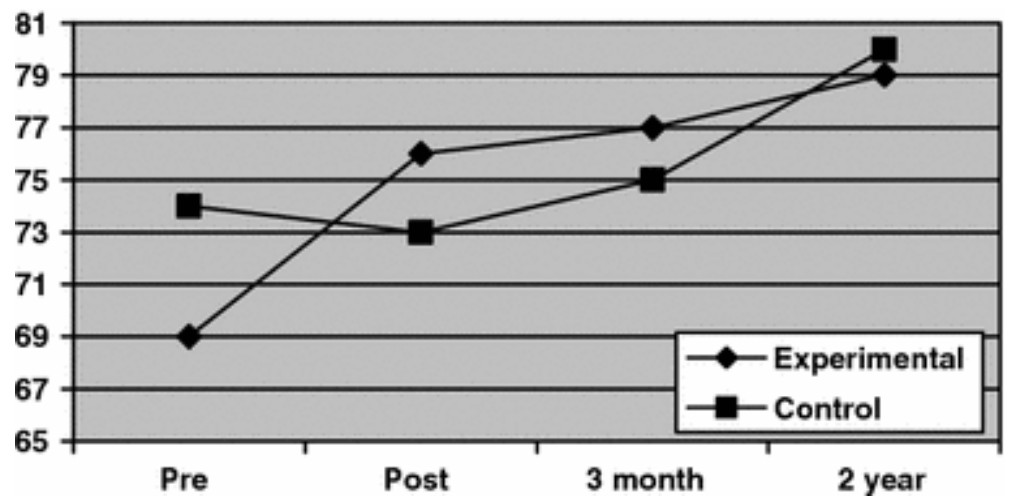

Fig. 2 Two year trajectories on the Comfort subscale of the KCAASS for experimental and control groups

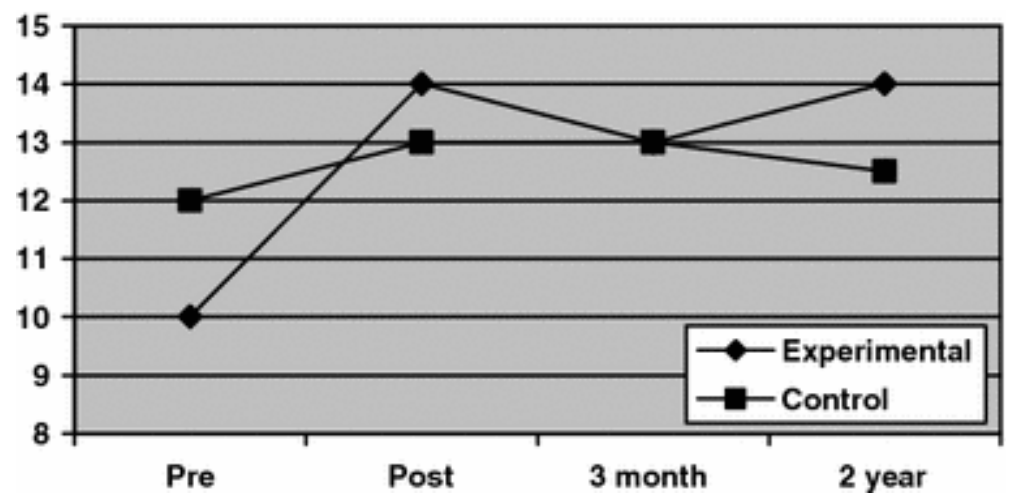

Fig. 3 Two year trajectories on the Approach subscale of the KCAASS for experimental and control groups 


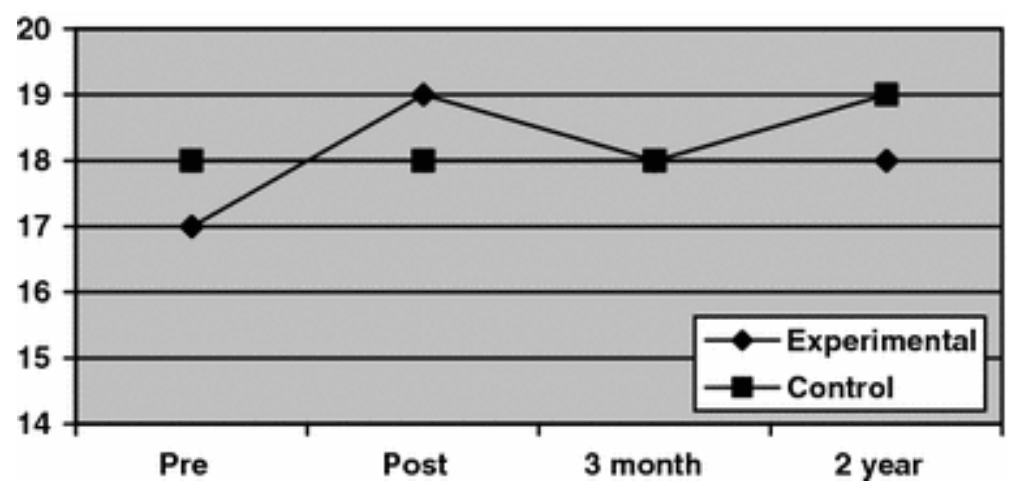

Fig. 4 Two year trajectories on the Attitude subscale of the KCAASS for experimental and control groups

Post hoc comparisons revealed that for the experimental group, 2 year follow-up scores for the Knowledge subscale were significantly higher than those at pre-training $(Z=-4.033, p<0.001)$ but did not differ significantly from scores at post-training and 3 month follow-up. A similar finding existed for the Comfort subscale $(Z=-3.321, p=0.001)$ and the Approach subscale $(Z=-3.319, p=0.001)$ with 2 year follow-up scores significantly higher than those at pre-training and no significant differences with those at post-training or 3 month follow-up. For the Attitude subscale, 2 year follow-up scores did not differ from pre-training or 3 month followup scores, but were significantly lower than those at post-training $(Z=-2.393, p=0.017)$.

Despite the finding that there was no significant change over the entire study period for the control group, it was found with post hoc comparisons that significant change occurred between 3 month follow-up and 2 year followup (pre and post control group training) on the Knowledge $(Z=-2.161, p=0.031)$ subscale. Post hoc comparisons also identified that significant change on the Attitude $(Z=-2.154, p=0.031)$ subscale occurred between the 3 month and 2 year follow-up (the period within which training occurred for this group). No other significant differences were noted.

Themes emerging from the qualitative data both support and extend the numerical findings. The first theme to emerge from the data was the benefits of the training, expressed in terms of the content material covered as well as within the multiple domains of impact.

The specific learning that occurred from the training was derived from multiple areas of material covered within the training including fertility, bladder and bowel management, relationships, erectile function, body image, emotional and psychological aspects of sexuality, contraception, positioning, counselling, medications, devices/sexual aids and communication.

Multiple domains of impact were similarly noted where in addition to knowledge acquisition, confidence, comfort and altered belief systems were engendered to address these issues and to support the translation of knowledge through to interactions with clients. 'Staff became more enthusiastic about the [sexuality] issue, wanting education and noticing differences in clients'. Practitioners suggested that sexual issues were more likely to be discussed with clients and that practitioners were more comfortable listening, more confident answering questions and more able to refer with confidence.

Attitudinal change towards the issue of sexuality was noted where previously it had been a topic that was not discussed. Now practitioners often 'put it in the same professional bracket as other things like bladder and bowel'. Another practitioner stated that 'I have a more open attitude about sex generally'. The training was described as 'desensitizing- so that you felt an increased comfort level when clients raised issues about sexuality'.

Further to this, some practitioners acknowledged that there was a process by which this influence occurred, with initial impacts on knowledge alone having consequential impacts on comfort and attitude over the longer term. 'Information improved our knowledge and then our confidence about issues and who to refer to'. As one practitioner stated: 
'The focus on sexuality has allowed me to be more informed about the issues which has in turn increased my confidence in response to questions'

A 'greater knowledge base engenders confidence' which was then identified as having flow on effects into other areas of professional practice such as practitioner/client/boundaries. Indeed, the training was identified as acting as a 'launch pad for other issues such as professional boundaries'.

The importance of context within which sexuality training occurs was highlighted as a major theme. Context was important in terms of the rehabilitation environment itself, the backgrounds, cultural beliefs and opinions of individual staff and the context of societal attitudes and assumptions. Each of these contextual factors influenced not only how practitioners learned, but also how comfortable they felt during the training, the extent to which attitudinal change could occur and be translated in clinical practice and the perceived benefits for the client in the longer term.

The rehabilitation environment for people with spinal cord injuries is unique in that individuals are part of that environment for a protracted period of time, during which they are dealing with multiple physical and psychological losses. Sexuality and sexual functioning is an important area where individuals (clients, family members and rehabilitation practitioners) perceive loss. As such, this environment and the issue of sexuality itself are emotionally charged. Practitioners identified both the 'emotionally intense context' and the 'emotionally sensitive issue'.

Similarly, individual backgrounds, cultural beliefs and value positions of practitioners contributed to the training in both positive and negative ways. Practitioners identified that 'there are strong opinions from different people with different backgrounds', referring to those practitioners who held fundamentalist Christian perspectives on sexuality and others from minority, culturally and linguistically diverse backgrounds. Training must therefore be inclusive and sensitive to different backgrounds, beliefs and values, while ensuring practitioners offer the same respect to colleagues and to individuals with spinal cord injuries who may also differ in their backgrounds, beliefs and opinions. Most importantly, training must develop in all practitioners the capacity to separate one's own personal value position from clinical interactions that aim to meet the needs of the client.

While considering the ways training had modified clinical practice, practitioners identified the broader contextual influences of societal attitudes and assumptions. They suggested that these attitudes and assumptions impacted on their perceived effectiveness in assisting clients to address sexuality issues and on the likely long-term outcomes of this assistance. Pragmatically, many practitioners identified that despite their perceived acceptance of sexuality among clients, this was not necessarily a view held by the general public. One practitioner said 'able-bodied people often feel uncomfortable around people with disabilities and won't talk to them because of their discomfort' and went onto say that 'most people would think a relationship with a person with SCI would be too hard and fraught with many inconveniences'. One practitioner captured this sentiment clearly, stating:

'I think anyone who doesn't conform to 'society's norm' for whatever reason will find it more difficult. It is not right but I believe in reality this is what happens'

Despite the contextual challenges within which the training occurred and the degree to which these challenges would continue to influence client sexuality in the long term, practitioners acknowledged that the training provided them with multiple benefits.

The mode of training was offered in a group setting and most practitioners identified the benefits of a group setting in normalizing issues and providing opportunities for debriefing, sharing experiences and problem solving. This was an important advantage of the training milieu, especially for the discussion of personal experiences. Further to this, the 'multidisciplinary approach' where disciplines were trained together was identified as particularly beneficial within a multidisciplinary rehabilitation setting. The link between the mode of training offered and the clinical context was important as it 'helped to understand views of others' and 'helped foster respect of other team members'. 
While the benefits of the group setting were recognized by participants, gaps in training that could address the nature of a constantly changing workforce were also identified. Continuing educational options such as self-directed learning packages were identified as one way to fill this gap. As one practitioner noted, 'there is quick turnover of staff yet we still have to deal with issues and new staff don't know what to do'. The issue of 'new staff and how do we train them' was identified as an area of concern by a number of practitioners. It was suggested that 'self directed learning packages are needed' and could be provided within a 'multidisciplinary inservice arena'. Further to this, others suggested a 'combination of learning options' was preferred while additional resources such as booklets, resource lists and yearly training updates were suggested. It was highlighted that training 'needs to be regularly repeated to maintain comfort and knowledge' for both new and more experienced practitioners. The importance of ongoing professional development in the rehabilitation setting through regular updates was proposed by practitioners who perceived a loss of some of their learnings over the 2 year period. 'In the 2 years since training, I have forgotten much of the content'. Another stated that 'it has been more difficult to consolidate the knowledge and skills from the training because I have not worked with people with SCI for the 2 years since the training'.

Further to the need for regular updates and in-services, a range of more advanced educational needs were identified even where practitioners felt that had gained the basics from the training. Topics identified by practitioners were fertility; products/devices for erectile function; counselling; practical ways of improving sexual functioning; psychosocial issues; medications; adolescent issues (first timers); contraception; positioning; relationships with partners and communication; impact of staff backgrounds and beliefs; visual aids; pregnancy; bladder and bowel; different sexual preferences; inappropriate behavior (individual and group); and professional boundaries.

\section{Discussion}

Sexuality training for healthcare practitioners has been conducted and evaluated in numerous healthcare arenas and such training has been beneficial to staff knowledge, comfort and attitudes in addressing client sexuality concerns [ $\underline{-1} \underline{13}]$. However, research that explores the long-term benefits of such training on practitioner knowledge, comfort, attitudes and behaviors has been lacking. In order to explore the long term benefits, this study was conducted as a 2 year follow-up to a randomized controlled trial of a sexuality training program for practitioners employed in spinal cord injury rehabilitation [ㅇ]. The study aimed to determine whether reported gains in the experimental group at 3 months were maintained at 2 years and whether the control group, who received training in the 4th month, had similarly improved knowledge, comfort and attitudes at the 2 year period.

As hypothesized, there were no significant between-group differences in knowledge, comfort or attitudes at 2year follow-up, indicating that both experimental and control groups had similar levels of knowledge, comfort and attitudes. This contrasts with the significant between-group differences previously reported at the 3-month follow-up [ $\underline{8}$ ] and highlights that the training of the control group at the 4 month period had resulted in equalisation of the groups. Although within-group changes were observed in the experimental group overall but not within the control group, post-hoc comparisons in the control group revealed significant changes in knowledge and attitude between 3 months and 2-year follow-up. So while there was no overall pattern of change for the control group, the period post-training for this group did demonstrate significant changes in knowledge and attitude, supporting the effectiveness of training.

The trend for higher scores on the Knowledge, Comfort and Attitude subscales within the control group at 2-year follow-up suggests a possible recency effect of training (e.g., scores are higher when measured closer to the training). This recency effect is supported by the fact that there was a small but insignificant decrease in knowledge and attitude scores between the measurement taken post-training and the 2 year-follow-up in the experimental group.

Failure to find an overall pattern of change over time in the control group is disappointing and may be related to higher initial scores exhibited by this group. It was previously suggested [ $\underline{8}$ ] that some resentment may have been 
evident within the control group due to their exclusion from the initial round of training, thereby artificially inflating their original questionnaire scores. After the delivery of training to the experimental group, practitioners from experimental and control groups were in working contact which may also be a contributing factor. A contamination effect had been anticipated where the control group may have improved knowledge through their interaction with the experimental group. While there was a trend for the control group to demonstrate increased knowledge scores in the period following the training of the experimental group, suggesting some transfer of knowledge, this was not statistically significant and is small when compared to changes in knowledge that occurred between the 3 month and 2-year period during which the control group were trained in the 4th month. This finding suggests that any knowledge transfer between practitioners does not provide the full benefits realised upon training provision.

Sexuality has traditionally been peripheral to the rehabilitation process and evidence supports that this hiatus continues in many areas [23]. However if we accept that sexuality is integral to the individual, then it follows sexuality needs to be considered in the delivery of holistic care [24]. In order to achieve this, practitioners must not only possess knowledge of sexuality issues, but must also feel comfortable in addressing them with clients. Comfort demonstrated a rising trend over the 2 year period in both experimental and control groups with the steepest increases evident following the provision of training. This contrasts with the downward trends noticed over time in knowledge. Despite differing starting points, both groups showed similar comfort levels at 2 years and these scores were higher than those exhibited prior to training. This supports the effectiveness of the training in improving comfort and suggests that comfort levels increase over time as practitioners utilise gains made in training in the practice setting.

This finding is supported by the analysis of qualitative data. Practitioners stated that training desensitized and helped to normalize sexuality as a client issue. This allowed them to treat sexuality in a similar fashion to other aspects of rehabilitation such as bladder, bowel or pain management. This finding is supported by Dixon-Woods et al. [25] who suggest that staff comfort can be improved by desensitisation. Therefore, the more exposure that practitioners have to discussions of sexual issues and the more they are able to locate this in the practice setting, the greater their comfort levels will become.

While stable and perhaps anticipated trends were noticed within groups for knowledge and comfort, scores on the Approach subscale were somewhat less predictable across both experimental and control groups. The analysis of qualitative data did not shed any light on the training benefits for managing comfort with personal approaches from clients. Personal approaches represent one of the most challenging aspects of managing sexual concerns among clients and are least likely to be discussed within the context of group training. The confronting nature of personal approaches may require more individualized support than a 1-day training program can provide. Mentoring, management support, debriefing and supervision may provide additional avenues to address these concerns while future research should be aimed at exploring and measuring interventions in this area.

Significant change over time was noted in both the experimental and control groups on the Attitude subscale. This is an important finding given that attitudinal change is more difficult to achieve than changes in comfort and knowledge [11]. Indeed, Dixon-Woods et al. [25] found that only 35\% of students felt their attitudes changed following training while changes in comfort and knowledge were more significant. Changes in attitude in the current study were significant but small. This may have been an artefact of the scale itself. Indeed, a change of one point on this scale could be clinically significant such as the difference between disagreeing or agreeing with the statement that 'people with spinal cord injuries should not worry about sex because they cannot feel it anyway'.

The small but significant changes in the numerical data on the Attitude subscale were supported by the qualitative findings that suggested attitudinal changes were related to increased knowledge and comfort. McKelvey et al. [26] similarly found knowledge was related to attitude with more negative attitudes reflected by lower levels of knowledge, especially towards more controversial topics among medical and nursing students. Inadequate knowledge is a recognized barrier to the acquisition of skills [17].

Indeed, Simpson et al. [13] proposed that knowledge and skills were more important aspects of training because 
attitudes were already fairly liberal and therefore desensitisation was unnecessary. The current findings, however, indicate that this may not necessarily be the case in all instances. Some practitioners reported a failure to challenge discriminatory attitudes despite disagreeing with them as these attitudes reflected those held by the general public. As rehabilitation practitioners are expected to role model appropriate attitudes towards disability, this tension highlights the importance of continuing to address attitudinal issues in training. Simpson et al. [13] identified training needs in a sample of community staff only compared to this study where the sample included hospital and community based practitioners of varying levels of experience. As a non-judgmental environment is imperative when discussing sexuality issues, it is essential that practitioners across the continuum of care recognize, acknowledge and develop awareness of prejudice and bias [17].

The qualitative findings highlight the importance of context with regard to prejudice and bias. The nature of context has implications for practitioner training, the effectiveness of such training in the rehabilitation setting, and the degree to which practitioners can translate what they have learned into practice. Although this study did not assess practitioner behavior directly, reports from participants indicated that some behavioral changes were translated into practice. Lewis and Bor [17] suggested that there are influences beyond knowledge on practitioner behavior that impact on attitude and have practical implications for rehabilitation environments. They found, for example, that ward scheduling and insufficient private spaces where sexuality concerns could be discussed were impediments in primary rehabilitation settings. This is consistent with findings from this study.

Guthrie [24] discussed issues such as practitioner perceptions of sexuality; reluctance to initiate discussion about sexuality with patients; stereotypical views of different sexual orientations; and the employment of avoidance coping strategies to manage their lack of time, heavy workload, and lack of privacy. He described the use of tactics such as avoiding, distancing, routinizing (behavior breaches) and humoring used by practitioners to manage embarrassment in ward contexts. Where the majority of time and attention is directed towards complex medical and functional issues, difficulties in addressing sexuality that can be time consuming is highlighted, again emphasising the importance of context [르. Factors such as the rehabilitation environment, practitioner backgrounds, cultural and value positions, and societal attitudes and assumptions were relevant to the training designed to address sexuality issues in this study. Each of these factors influenced both positively and negatively, how practitioners learned, how comfortable they felt during the training, the degree of openness to attitudinal change, the willingness to translate gains into clinical practice and the perceived benefits for the client in the longer term.

Interprofessional learning contexts are under-utilised in rehabilitation settings where training for the rehabilitation team is more commonly conducted within discipline-specific silos [28]. This approach fails to realise the potential benefits of interprofessional training. It fosters team work, enables practitioners to understand that responses to sexuality issues are not limited to 'other disciplines' and that practitioners gain from the experiences of their colleagues. The analysis of qualitative data supported the benefits of the interdisciplinary learning contexts. Despite challenges providing training relevant to different disciplinary groups, levels of experience and background, the multiple benefits that participation provided were identified. One such issue was an improved understanding that sexual issues are raised with all team members regardless of discipline. It also assisted medical and allied health practitioners to recognize how the intimate nature of the nurse-patient relationship, places nurses in the ideal position to address these issues in the first instance [17]. Practitioners also highlighted the importance of the group setting for training as beneficial to the discussion of personal experiences previously identified by Dixon-Woods et al. [25] who found that group work allows individuals to compare their values and attitudes with others in a way that would not be possible in a formal lecture setting.

Continuing professional development opportunities in sexuality rehabilitation were identified as important to those practitioners who reported a decrease in knowledge and comfort because they lacked opportunity to utilise the skills learned in the 2 years following their training. The experiential notion of 'use it or lose it' combined with staff turnover and the orientation of new staff reinforced the need to develop continuing educational options. This may be particularly important where skill mix and differences in experiences between practitioners are evident. While there are opportunities for informal knowledge transfer and increased comfort in an environment where sexual matters are openly discussed, more formal mechanisms may be required to ensure that knowledge, comfort and attitudes related to sexuality are addressed adequately, particularly in relation to personal approaches 
where additional supports such as mentoring and supervision may apply. Indeed, in relation to continuing education, practitioners identified rehabilitation, of which sexuality is a part, as constantly subjected to change thus creating the need for updating on new techniques on a regular basis.

\section{Limitations}

There are a number of limitations to this study. These include the smaller numbers in the control group at the 2year follow-up period that may have contributed to differences identified between experimental and control groups. Although there was less missing data at the follow-up period, the numbers lost to attrition were high and this was especially evident with nursing staff within the control group. While there were no significant differences in KCAASS scores at any measurement point between individuals in the follow-up group and those lost to attrition (in either the experimental or control group), the remaining 12 individuals within the control group at 2 year follow-up were unique in discipline and may have differed on other unmeasured characteristics. Future trials should explore the use of placebo training to prevent attrition that may be the result of negativity within control groups.

The field context of the single rehabilitation service creates its own issues for conducting randomized trials such as contamination between experimental and control groups and social desirability biases (e.g. negativity in control group). Another example of the impact of field context lies in the fact that the qualitative data was collected through a focus group conducted prior to administration of the 2 year follow-up KCAASS for logistic reasons. Although there was a 4 month period separating the two modes of data collection, there was the possibility that the focus group may have responded differently to the follow-up questionnaire on the basis of information discussed within the focus group discussion rather than on actual long-term changes in knowledge, comfort and attitude.

The lack of sensitivity of the Attitude subscale of the KCAASS may have presented challenges to the accurate capture of attitudinal change while time and cost prevented the use of observational methods to measure behavioral change, significantly limiting the degree to which translational benefits of the training could be understood. Finally, the training was developed and evaluated on the basis of individualized training needs that were assessed at the commencement of the initial randomized controlled trial. As such, the degree to which the current findings can be generalized to other groups is potentially limited.

\section{Implications for Practice}

Recommendations for clinical practice and future research that emerge from this study stem from the benefits of providing training that is individualized to the needs of the consumer. Perhaps the long-term benefits of the training were related to its development from a needs assessment. The topics it covered were determined by the consumers rather than experts. Comparative studies on different training modalities would help to disentangle these effects.

Future research needs to be conducted to determine the degree to which these changes may directly impact on behavior and practice. Clinically, ongoing support to practice and the development of action plans using training content and adaptation to real situations are warranted. In order to support practitioner management of personal approaches, other strategies such as parallel mentoring and peer support systems for new staff may be particularly useful.

Exploration and evaluation of continuing educational options such as booklets or lists of resources that can be accessed to refresh knowledge in addition to refresher courses would be helpful. Interventions that address more advanced educational needs, offering extended training on fertility, counselling, contraception, medications, professional boundaries and the impact of practitioner backgrounds and beliefs are also indicated. 


\section{Conclusions}

This 2-year follow-up on the provision of sexuality training for rehabilitation professionals working in spinal cord injury rehabilitation has demonstrated that training can have long-lasting effects on practitioner knowledge, comfort and attitudes in addressing sexuality issues with their clients. Education that is developed around the individualized needs of practitioners and the settings within which they work may be particularly effective. Future research should be used to explore whether these approaches are applicable in other rehabilitation and healthcare settings; to explore the most effective interventions to assist practitioners in the management of personal sexual approaches; and to measure how knowledge is translated from training to the practice setting.

\section{References}

1. McAlonan, S.: Improving sexual rehabilitation services: the patient's perspective. Am. J. Occup. Ther. 50, 826-834 (1996)

2. Haboubi, N.H., Lincoln, N.: Views of health professionals on discussing sexual issues with patients. Disabil. Rehabil. 25 291-296 (2003)

3. Dunn, M.: Sexual questions and comments on a spinal cord injury service. Sex. Disabil. 6, 126-134 (1983)

4. Herson, L., Hart, K.A., Gordon, M.J., Rintala, D.H.: Identifying and overcoming barriers to providing sexuality information in the clinical setting. Rehabil. Nurs. 24, 148-151 (1999)

5. Lemon, M.A.: Sexual counselling and spinal cord injury. Sex. Disabil. 11, 73-97 (1993)

6. Summerville, P., McKenna, K.: Sexuality education and counselling for individuals with a spinal cord injury: implications for occupational therapy. Br. J. Occup. Ther. 61, 275-279 (1998)

7. Tepper, M.S.: Providing comprehensive sexual health care in spinal cord injury rehabilitation: implementation and evaluation of a new curriculum for health care professionals. Sex. Disabil. 15, 131-165 (1997)

8. Fronek, P., Booth, S., Kendall, M., Miller, D., Geraghty, T.: The effectiveness of a sexuality training program for the interdisciplinary spinal cord injury rehabilitation team. Sex. Disabil. 23, 51-63 (2005)

9. Chivers, J., Mathieson, S.: Training in sexuality and relationships: an Australian model. Sex. Disabil. 18, 73-80 (2000)

10. Rose, J., Holmes, S.: Changing staff attitudes to the sexuality of people with mental handicaps and evaluative comparison of one and three day workshops. Ment. Hand. Res. 4, 67-79 (1991)

11. Walker, B., Harrongton, D.: Effects of staff training on staff knowledge and attitudes about sexuality. Educ. Gerontol. 28 , 
12. Post, M.W.M., Gianotten, W.L., Heinen, L., Hille Ris Lambers, E., Willems, M.: Sexological competence of different rehabilitation disciplines and effects of a discipline-specific sexological training. Sex. Disabil. 26, 3-14 (2008)

13. Simpson, G., Anwar, S., Wilson, J., Bertapelle, T.: Improving the rehabilitative management of client sexual health concerns after neurological disability: evaluation of a staff sexuality training program in New Zealand. Clin. Rehabil. 20 , 847-859 (2006)

14. Couldrick, L.: Sexual issues within occupational therapy: part 2: implications for education and practice. Br. J. Occup. Ther. 62, 26-30 (1999)

15. Hodge, A.L.: Addressing issues of sexuality with spinal cord injured persons. Ortho. Nurs. 14, 21-24 (1995)

16. Couldrick, L.: Sexual issues within occupational therapy: part 1: attitudes and practice. Br. J. Occup. Ther. 61, 538-544 (1998)

17. Lewis, S., Bor, R.: Nurses' knowledge of and attitudes towards sexuality and the relationship of these with nursing practice. J. Adv. Nurs. 20, 251-259 (1994)

18. Kendall, M., Booth, S., Fronek, P., Miller, D., Geraghty, T.: The development of a scale to assess the training needs of professionals in providing sexuality rehabilitation following spinal cord injury. Sex. Disabil. 21, 49-64 (2003)

19. Booth, S., Kendall, M., Fronek, P., Miller, D., Geraghty, T.: Training the interdisciplinary rehabilitation team in sexuality rehabilitation following spinal cord injury: a needs assessment. Sex. Disabil. 21, 249-261 (2003)

20. Kendall, M., Ungerer, G., Dorsett, P.: Bridging the gap: transitional rehabilitation services for people with spinal cord injury. Disabil. Rehabil. 25, 1008-1015 (2003)

21. Aronson, J.: A pragmatic view of thematic analysis. The Qualitative Report, vol. 2, Accessed October 21, 2009 from http://www.nova.edu/ssss/QR/Backlssues/QR2-1/aronson.html. (1994)

22. Taylor, S.J., Bogdan, R.: Introduction to Qualitative Research Methods: The Search for Meanings. Wiley, New York (1984)

23. Wazakili, M., Mpofu, R., Devlieger, P.: Should issues of sexuality and HIV and AIDS be a rehabilitation concern? The voices of young South Africans with physical disabilities. Disabil. Rehabil. 31(1), 32-41 (2009)

24. Guthrie, C.: Nurses' perceptions of sexuality relating to patient care. J. Clin. Nurs. 8, 313-321 (1999) 
25. Dixon-Woods, M., Regan, J., Robertson, N., Young, B., Cordle, C., Tobin, M.: Teaching and learning about human sexuality in undergraduate medical education. Med. Educ. 36, 432-440 (2002)

26. McKelvey, R.S., Webb, J.A., Baldassar, L.V., Robinson, S.M.: Sex knowledge and sexual attitudes among medical and nursing students. Aust. N. Z. J. Psychiatry 33, 260-266 (1999)

27. Murphy, N.A., Elias, E.R.: Sexuality of children and adolescents with developmental disabilities. Pediatrics 118(1), 398403 (2006)

28. Fronek, P., Kendall, M., Ungerer, G., Malt, J., Eugarde, E., Geraghty, T.: Towards healthy professional-client relationships: the value of an interprofessional training course. J. Interprof. Care 23, 16-29 (2009) 